\title{
Convective Instability of Magnetized Ferrofluids: Influence of Magnetophoresis and Soret Effect
}

\author{
Mark I. Shliomis \\ Department of Mechanical Engineering, Ben-Gurion University of the Negev, \\ P.O.B. 653, Beer-Sheva 84105, Israel
}

\begin{abstract}
Convective instability in a ferrofluid layer heated from below or from above in the presence of a uniform vertical magnetic field is investigated theoretically. Convection is caused by a magnetic mechanism based on the temperature and concentration dependence of magnetization. An imposed temperature gradient establishes (by the Soret effect) a concentration gradient of magnetic particles of which the ferrofluid is composed. Both these gradients cause a spatial variation in magnetization, which induces a gradient of magnetic field intensity within the fluid layer. The field gradient induces in its turn an additional redistribution of magnetic grains due to magnetophoresis. Resulting self-consistent magnetic force tries to mix the fluid. A linear stability analysis predicts oscillatory instability in a certain region of the magnetic field strength and the fluid parameters. The instability owes magneto- and thermophoresis its origin: were the particle diffusion not operative, then only stationary instability would occur. A discovery of predicted convective oscillations is expected in ferrofluid layers about $1 \mathrm{~mm}$ thick, where the buoyancy mechanism is negligible and the characteristic diffusion time is not too long.
\end{abstract}

\section{Introduction}

Magnetic colloids (so-called ferrofluids $[1,2]$ ) represent very interesting object for studies of convection. Even in the absence of the gravity, mechanical equilibrium of a nonisothermal ferrofluid in a magnetic field $H$ is in general impossible. At the basis of the mechanism of thermomagnetic convection [1-5] lies the temperature dependence of the magnetization $M$ : under otherwise equal conditions, the colder elements of the fluid are more strongly magnetized, and therefore they are subject to a larger magnetic force in the direction of $\nabla H$. The gradients of the magnetic field intensity here play the same role as does the gravitational field $\mathbf{g}$ in the mechanism of ordinary thermogravitational convection, whilst the temperature dependence of the magnetization, $M(T)$, plays the role of $T$-dependence of the fluid density $\rho$.

Interestingly, thermomagnetic convection can arise even in a uniform applied magnetic field [4]. Let a ferrofluid confined between two horizontal planes be in a uniform vertical external field $\mathbf{H}_{\mathrm{e}}=\left(0,0, H_{\mathrm{e}}\right)$ at the temperature $T(z)$. Then the dependence $M(T)$ leads to the result that the field inside the fluid, $\mathbf{H}=(0,0, H)$, appears to be nonuniform. Indeed, from the Maxwell equation $\operatorname{div} \mathbf{B}=0$ and the definition of magnetic induction $\mathbf{B}=\mathbf{H}+4 \pi \mathbf{M}$ it follows

$$
\frac{\mathrm{d} H}{\mathrm{~d} z}=-4 \pi \frac{\mathrm{d} M}{\mathrm{~d} z}
$$


that yields

$$
\frac{\mathrm{d} H}{\mathrm{~d} z}=-\frac{4 \pi}{\hat{\mu}}\left(\frac{\partial M}{\partial T}\right)_{\mathrm{H}} \frac{\mathrm{d} T}{\mathrm{~d} z},
$$

where $\hat{\mu}=1+4 \pi(\partial M / \partial H)$ is the differential magnetic permeability. Equation (1.1) shows that the induced gradient of magnetic field is directed always opposite to the gradient of magnetization. It results in unstable magnetization stratification: the magnetic force $\mathbf{F}=M \nabla H$ tries to mix ferrofluid. According to eq. (1.2), this force is proportional to the imposed temperature gradient. Therefore, when both the magnetic and buoyancy mechanisms are operative, the first of two predominates over the second one at the onset of convection in a thin enough fluid layers, where the critical temperature gradient is sufficiently large. One can show [1] that the condition

$$
\left|\left(\frac{\partial M}{\partial T}\right)_{\mathrm{H}} \frac{\mathrm{d} H}{\mathrm{~d} z}\right| \gg\left|\left(\frac{\partial \rho}{\partial T}\right)_{\mathrm{p}} g\right|,
$$

which permits neglect the buoyancy mechanism of convection, is reduced to

$$
d^{4} \ll 4 \pi R a_{\mathrm{c}} \eta \kappa\left[\frac{(\partial M / \partial T)_{\mathrm{H}}}{g(\partial \rho / \partial T)_{\mathrm{p}}}\right]^{2},
$$

where $d$ is the layer thickness, $R a_{\mathrm{c}} \sim 10^{3}$ the critical Rayleigh number $\left(R a_{\mathrm{c}}=\right.$ 1708 in the case of rigid boundaries and $R a_{\mathrm{c}}=27 \pi^{4} / 4 \approx 657.5$ for free boundaries), $\eta$ and $\kappa$ the fluid viscosity and thermodiffusivity. For commonly used magnetite ferrofluids on the base of water or kerosene the pyromagnetic coefficient $-(\partial M / \partial T)_{\mathrm{H}}$ in the field $H \sim 200-500$ Oe is about $0.5 \phi \mathrm{G} / \mathrm{K}$ where $\phi$ is the volume fraction of magnetic grains. Then for $\phi \sim 0.1, \eta \sim 10^{-2} \mathrm{Ps}, \kappa \sim$ $10^{-3} \mathrm{~cm}^{2} / \mathrm{s}$, and $-(\partial \rho / \partial T)_{\mathrm{p}} \sim 5 \times 10^{-4} \mathrm{~g} \mathrm{~cm}^{-3} \mathrm{~K}^{-1}$, the inequality (1.3) holds true down to $d=1 \mathrm{~mm}$. The same conclusion has been done by Finlayson [4] who first studied theoretically convective instability of a ferrofluid layer heated from below in the presence of a uniform vertical magnetic field.

It is important to notice that in [4] and posterior theoretical works [5-8] ferrofluids were treated as pure (i.e., single-component) magnetized fluids. Such a model assumes a fixed uniform concentration of magnetic grains, $\phi=$ const. Ignoring in that way diffusion effects, authors of [4-8] came to conclusion that only stationary convective instability is possible in ferrofluids. As it pointed out already in the abstract of Ref. [4], "oscillatory instability cannot occur". But a real ferrofluid should be treated as a binary mixture with allowance for concentration dependence of its magnetization. An externally imposed temperature gradient induces in a ferrofluid layer a concentration gradient owing to the Soret effect. Both these gradients cause a spatial variation in magnetization $M(\phi, T, H)$ that leads in turn to appearance of a magnetic field gradient within the fluid layer. Thus there arise magnetic forces, which try to mix the ferrofluid and, in addition, they induce a certain redistribution of magnetic grains due to 
magnetophoresis. As it will be shown below, just the magnetophoresis together with the thermophoresis (the Soret effect) give rise to oscillatory instability in a certain region of the magnetic field intensity and the fluid parameters. Appropriate experimental conditions to observe predicted instability take place in a thin ferrofluid layer, when the ordinary (buoyancy) mechanism of convection is negligible and the characteristic diffusion time, $\tau_{\mathrm{D}}=d^{2} / \pi^{2} D$, required to settle a steady state, is not too long (here $D$ is the diffusion coefficient of magnetic particles in the basic liquid). Recent measurements [9] yielded $D=2.7 \times 10^{-7} \mathrm{~cm}^{2} / \mathrm{s}$ in water and cyclohexane. (This magnitude of $D$ agrees well with a theoretical estimate by the Einstein's formula for the diffusion coefficient, $D=k_{\mathrm{B}} T / 6 \pi \eta a$, where $2 a \simeq 10 \mathrm{~nm}$ is the diameter of a magnetic grain). According to above formula for $\tau_{\mathrm{D}}$, the concentration equilibrium in a ferrofluid layer of the thickness $d=1 \mathrm{~mm}$ is reached after one hour.

In the next Section we obtain the description of the magnetophoresis and Soret effect in a ferrofluid assuming that the fluid behaves with respect to an external magnetic field like a Langevin's paramagnet $[1,10]$. In Secs. 3 and 4 we derive the complete set of modified linear equations for perturbations and settle the boundary conditions for them. The eigenvalue problem is solved in Sec. 5 for the Bénard configuration. On the base of the solution, oscillatory instability is predicted to occur for a wide range of magnitudes of magnetic field, Soret coefficient, and other fluid parameters.

Owing to the small magnitude of $D$ (see above), there are possible two scenarios of the beginnings of convection. Depending on the imposed heating rate, the ferrofluid may behave either as a binary mixture or like a pure fluid. Both the possibilities are discussed in Sec. 6 .

\section{Magnetophoresis and Soret Effect}

In a nonuniform magnetic field there acts on each particle a force $\mathbf{f}=(\mathbf{m} \nabla) \mathbf{H}$. Orientation of the particle magnetic moment $\mathbf{m}$ in the direction of $\mathbf{H}$ is impeded by thermal motion. As a result, the mean value of $\mathbf{m}$ appears to be equal to

$$
\langle\mathbf{m}\rangle=m \mathcal{L}\left(\frac{m H}{k_{\mathrm{B}} T}\right) \mathbf{e}, \quad \mathbf{e}=\frac{\mathbf{H}}{H}, \quad \mathcal{L}(\xi)=\operatorname{coth} \xi-\xi^{-1} .
$$

With allowance for the identity $\mathbf{H} \times \operatorname{rot} \mathbf{H}=\frac{1}{2} \nabla H^{2}-(\mathbf{H} \nabla) \mathbf{H}$ and the condition of absence of electric currents in nonconducting ferrofluids, $\operatorname{rot} \mathbf{H}=\mathbf{0}$, formula (2.1) enable us to write the mean magnetophoretic force $\langle\mathbf{f}\rangle=m \mathcal{L}(\xi) \nabla H$. This force and the Stokes drag coefficient, $6 \pi \eta a$, for a sphere of the radius $a$ determine the regular component of the Brownian velocity of the particle with respect to the liquid,

$$
\mathbf{u}=\frac{m}{6 \pi \eta a} \mathcal{L}(\xi) \nabla H
$$

The volume density of the diffusion flux of the matter in a ferrofluid may be written

$$
\mathbf{j}=\phi \mathbf{u}-D\left(\nabla \phi+S_{\mathrm{T}} \nabla T\right)
$$


where $S_{\mathrm{T}}$ is the Soret coefficient. Substituting (2.2) in (2.3) and using the Einstein's formula for the diffusion coefficient, we obtain

$$
\mathbf{j}=-D\left[\nabla \phi+S_{\mathrm{T}} \nabla T-(\phi / H) \xi \mathcal{L}(\xi) \nabla H\right]
$$

Thus the matter flux is provided by the "diffusiophoresis" ( $\propto \nabla \phi$; this term was proposed by Derjaguin et al. [11]), thermophoresis $(\propto \nabla T)$, and magnetophoresis $(\propto \nabla H)$. If, however, confined horizontal planes are impervious and an applied magnetic field is uniform, then gradients of $\phi$ and $H$ can be caused only by an imposed temperature gradient. Therefore in the steady state $(\mathbf{j}=0)$ the values of $\nabla \phi$ and $\nabla H$ are expressed via $\nabla T$. As it is seen from (2.4), the equilibrium equation $j_{\mathrm{z}}=0$ reads

$$
\frac{\mathrm{d} \phi}{\mathrm{d} z}=-S_{\mathrm{T}} \frac{\mathrm{d} T}{\mathrm{~d} z}+\phi \xi \mathcal{L} \frac{\mathrm{d} \ln H}{\mathrm{~d} z} .
$$

Substituting in (1.1) $M=\phi M_{\mathrm{S}} \mathcal{L}\left(m H / k_{\mathrm{B}} T\right)$ where $M_{\mathrm{S}}$ is the saturation magnetization of the particle material, we find

$$
\hat{\mu} \frac{\mathrm{d} H}{\mathrm{~d} z}=-4 \pi\left(\frac{\mathrm{d} \ln \phi}{\mathrm{d} z}-\frac{\xi \mathcal{L}^{\prime}}{\mathcal{L}} \frac{\mathrm{d} \ln T}{\mathrm{~d} z}\right) .
$$

Eliminating now the concentration gradient from (2.5) and (2.6), we arrive at

$$
\frac{\mathrm{d} H}{\mathrm{~d} z}=\frac{4 \pi M}{\sigma T}\left(\Psi+\frac{\xi \mathcal{L}^{\prime}}{\mathcal{L}}\right) \frac{\mathrm{d} T}{\mathrm{~d} z},
$$

where $\sigma=\hat{\mu}+12 \pi \chi_{0} \mathcal{L}^{2}(\xi)=1+12 \pi \chi_{0}\left(\mathcal{L}^{\prime}+\mathcal{L}^{2}\right), \chi_{0}=\phi M_{\mathrm{s}} m / 3 k_{\mathrm{B}} T$ the initial Langevin magnetic susceptibility, and $\Psi=(T / \phi) S_{\mathrm{T}}$ plays the role of the separation ratio in the mechanism of thermomagnetic convection. Substituting $\mathrm{d} H / \mathrm{d} z$ from (2.7) into (2.5) we obtain, finally,

$$
\frac{\mathrm{d} \phi}{\mathrm{d} z}=-\frac{\hat{\mu} \phi}{\sigma T}\left[\Psi-\frac{(\hat{\mu}-1)}{\hat{\mu}} \xi \mathcal{L}\right] \frac{\mathrm{d} T}{\mathrm{~d} z} .
$$

One can rewrite the latter in the form

$$
\frac{\mathrm{d} \phi}{\mathrm{d} z}=-\frac{\phi}{T} \Psi_{\mathrm{H}} \frac{\mathrm{d} T}{\mathrm{~d} z},
$$

introducing the field-dependent separation ratio

$$
\Psi_{\mathrm{H}}=\frac{\hat{\mu}}{\sigma}\left[\Psi-\frac{(\hat{\mu}-1)}{\hat{\mu}} \xi \mathcal{L}\right]
$$

Note that $\Psi_{\mathrm{H}}$ differs from zero even if $\Psi=0$, i.e., in the absence of the Soret effect. The non-Soret $\phi-T$ coupling originates from the magnetophoresis, which is manifested as a negative separation ratio:

$$
\left.\Psi_{\mathrm{H}}\right|_{\Psi=0}=-(\hat{\mu}-1) \xi \mathcal{L} / \sigma<0
$$


Actually, the induced gradient of magnetic field (2.7) is directed along the temperature gradient, thus the hotter fluid (and hence the stronger magnetic field) - the higher concentration of magnetic grains. By the same manner it behaves a nonmagnetic binary mixture with a negative separation ratio. The function defined by Eq. (2.11) is proportional to $\xi^{2}$ at $\xi \ll 1$ and inversely proportional to $\xi$ at $\xi \gg 1$. For a ferrofluid with the initial magnetic permeability $\mu_{0}=1+4 \pi \chi_{0}=3$ this function has a distinct minimum, $\left.\Psi_{\mathrm{H}}\right|_{\Psi=0}=-0.303$, at $\xi=2.30$.

\section{Equations for Stability Analysis}

The general expression for the volume density of magnetic force acting a nonconducting and incompressible ferrofluid may be written [12]

$$
\mathbf{F}=\nabla\left\{\frac{H^{2}}{2}\left[\rho\left(\frac{\partial \chi}{\partial \rho}\right)_{\mathrm{T}, \mathrm{H}}-\chi\right]\right\}+M \nabla H,
$$

where $\chi=M / H$ stands for the magnetic susceptibility. The first term in (3.1) is known as the magnetostrictive force and the second one called the Kelvin force. Under the substitution $\mathbf{F}$ in the equation of fluid motion, the magnetostrictive force which originates from a potential is included in the pressure gradient, $\nabla p$, so this force is equilibrated automatically. Thus it remains only the Kelvin force, which in equilibrium should be also balanced at each point by the pressure gradient: $\nabla p=M \nabla H$. On applying the operation rot to this equation, we obtain a necessary condition for equilibrium,

$$
\left(\frac{\partial M}{\partial T} \nabla T+\frac{\partial M}{\partial \phi} \nabla \phi\right) \times \nabla H=0 .
$$

In the case under consideration, all three gradients contained in (3.2) are parallel to each other (they have only $z$-components), so the condition (3.2) is satisfied. Thus, the equilibrium is possible, and we set about a study of its stability.

Small perturbations of a standing mode will be characterized by velocity $\mathbf{v}$, pressure $p$, temperature $\theta$, concentration $\varphi$, and magnetic field $\mathbf{h}$. Then the perturbation $\mathbf{F}^{\prime}$ of the magnetic force density $\mathbf{F}=M \nabla H$ is expressed via $\theta, \varphi$ and h:

$$
\mathbf{F}^{\prime}=-h_{\mathrm{z}} \nabla M+\left(\frac{\partial M}{\partial T} \theta+\frac{\partial M}{\partial \phi} \varphi+\frac{\partial M}{\partial H} h_{\mathrm{z}}\right) \nabla H .
$$

[We have omitted here the term $\nabla\left(M h_{\mathrm{z}}\right)$. Later on it is assumed to be included in the gradient of the pressure perturbation, $\nabla p$, see Eq. (3.6)]. In the equilibrium $\nabla M$ and $\nabla H$ are directed along the $z$ axis; so, using (1.1) we obtain from (3.3) the only component of the vector $\mathbf{F}^{\prime}$ :

$$
F_{\mathrm{z}}^{\prime}=\left(\frac{\hat{\mu}}{4 \pi} h_{\mathrm{z}}+\frac{\partial M}{\partial T} \theta+\frac{\partial M}{\partial \phi} \varphi\right) \frac{\mathrm{d} H}{\mathrm{~d} z} .
$$


Substituting in the formula $M=\phi M_{\mathrm{s}} \mathcal{L}(\xi), \xi=m H / k_{\mathrm{B}} T$, and $\mathrm{d} H / \mathrm{d} z$ from (2.7), we get

$$
F_{\mathrm{z}}^{\prime}=\frac{M}{\sigma T}\left(\Psi+\frac{\xi \mathcal{L}^{\prime}}{\mathcal{L}}\right)\left[\hat{\mu} h_{\mathrm{z}}+4 \pi M\left(\frac{\varphi}{\phi}-\frac{\xi \mathcal{L}^{\prime}}{\mathcal{L}} \frac{\theta}{T}\right)\right] \frac{\mathrm{d} T}{\mathrm{~d} z} .
$$

Thus, the linear equation of fluid motion takes the form

$$
\rho \frac{\partial \mathbf{v}}{\partial t}=-\nabla p+\eta \nabla^{2} \mathbf{v}+\frac{M \Delta T}{\sigma T d}\left(\Psi+\frac{\xi \mathcal{L}^{\prime}}{\mathcal{L}}\right)\left[4 \pi M\left(\frac{\varphi}{\phi}-\frac{\xi \mathcal{L}^{\prime}}{\mathcal{L}} \frac{\theta}{T}\right)+\hat{\mu} \frac{\partial \Phi}{\partial z}\right] \mathbf{e}
$$

We have substituted in (3.6) the magnitude of the stationary temperature gradient, $\mathrm{d} T / \mathrm{d} z=\Delta T / d$, where $\Delta T$ is the temperature difference between confined surfaces of the layer. In (3.6) $\mathbf{e}$ is the unit vector along the $z$ axis, and $\Phi$ the potential of magnetic field perturbations, $\mathbf{h}=\nabla \Phi$. To derive an equation for $\Phi$ one should substitute the perturbation of magnetic induction,

$$
\mathbf{b}=\mathbf{h}+4 \pi \frac{M}{H}\left(\mathbf{h}-h_{\mathrm{z}} \mathbf{e}\right)+4 \pi\left(\frac{\partial M}{\partial T} \theta+\frac{\partial M}{\partial \phi} \varphi+\frac{\partial M}{\partial H} h_{\mathrm{z}}\right) \mathbf{e}
$$

in the Maxwell equation $\operatorname{div} \mathbf{b}=0$. It yields

$$
\mu \nabla^{2} \Phi-(\mu-\hat{\mu}) \frac{\partial^{2} \Phi}{\partial z^{2}}=-4 \pi M\left(\frac{1}{\phi} \frac{\partial \varphi}{\partial z}-\frac{\xi \mathcal{L}^{\prime}}{T \mathcal{L}} \frac{\partial \theta}{\partial z}\right),
$$

where $\mu=1+4 \pi(M / H)=1+12 \pi \chi_{0} \mathcal{L}(\xi) / \xi$.

Equation (2.4) under the replacement $\phi \rightarrow \phi+\varphi, T \rightarrow T+\theta$, and $H \rightarrow$ $H+\partial \Phi / \partial z$ determines the matter flux perturbation. So, making allowance for Eq. (2.9), we obtain the diffusion equation

$$
\frac{\partial \varphi}{\partial t}=D \nabla^{2}\left(\varphi+\frac{\phi}{T} \Psi \theta-\xi \mathcal{L} \frac{\phi}{H} \frac{\partial \Phi}{\partial z}\right)+\frac{\phi \Delta T}{T d} \Psi_{\mathrm{H}}(\mathbf{v e}) .
$$

Equations (3.6)-(3.8) together with the heat conduction equation,

$$
\frac{\partial \theta}{\partial t}=\kappa \nabla^{2} \theta-(\Delta T / d)(\mathbf{v e})
$$

and the condition of incompressibility, $\operatorname{div} \mathbf{v}=0$, form the complete set of equations which determine the onset of convection in a magnetized ferrofluid.

Pass over to dimensionless variables by choosing a unit of length $d$, time $d^{2} / \kappa$, velocity $\kappa / d$, temperature $\Delta T$, concentration $(\Delta T / T) \phi$, and field potential $4 \pi M(\Delta T / T) d$. Then the above equations take the form

$$
\operatorname{Pr}^{-1} \frac{\partial \mathbf{v}}{\partial t}=-\nabla p+\nabla^{2} \mathbf{v}+R m G\left[\Omega-\left(\Psi+\frac{\xi \mathcal{L}^{\prime}}{\mathcal{L}}\right) \theta+\sigma \mathrm{D} \Phi\right] \mathbf{e}
$$




$$
\begin{gathered}
\frac{\partial \theta}{\partial t}=\nabla^{2} \theta-(\mathbf{v e}), \quad \operatorname{div} \mathbf{v}=0 \\
\frac{\partial \Omega}{\partial t}=L e \nabla^{2} \Omega+\Psi \frac{\partial \theta}{\partial t}-(\sigma-\hat{\mu}) \mathrm{D} \frac{\partial \Phi}{\partial t}+\Psi_{\mathrm{H}}(\mathbf{v e}) \\
\mu \nabla^{2} \Phi+(\sigma-\mu) \mathrm{D}^{2} \Phi=-\mathrm{D}\left[\Omega-\left(\Psi+\frac{\xi \mathcal{L}^{\prime}}{\mathcal{L}}\right) \theta\right], \quad \nabla^{2} \Phi_{\mathrm{e}}=0 .
\end{gathered}
$$

Here $\operatorname{Pr}=\eta / \rho \kappa$ is the Prandtl number, $L e=D / \kappa$ the Lewis number, $\mathrm{D} \equiv \mathrm{d} / \mathrm{d} z$,

$$
R m=\frac{\left[\phi M_{\mathrm{s}} \Delta T d\right]^{2}}{\eta \kappa T^{2}}, \quad G=\frac{4 \pi \mathcal{L}^{2}}{\sigma}\left(\Psi+\frac{\xi \mathcal{L}^{\prime}}{\mathcal{L}}\right),
$$

where $R m$ we shall call the magnetic Rayleigh number, $\Phi_{\mathrm{e}}$ is the potential of field perturbations outside the fluid layer, and $\Omega=\varphi+\Psi \theta-(\sigma-\hat{\mu}) \mathrm{D} \Phi$ is introduced as an independent variable instead of the concentration perturbation $\varphi$. It is worth to note that $\nabla \Omega$ has the physical meaning of the matter flux perturbation.

Studying the onset of convection in the Bénard configuration, one should put all perturbations be dependent on time and horizontal coordinates as $\exp [\lambda t+$ $\left.\imath\left(k_{\mathrm{x}} x+k_{\mathrm{y}} y\right)\right]$. Then from Eqs. (3.10) it follows the system of equations for $z$ dependent amplitudes of $z$-component of velocity $W=(\mathbf{v e})$, temperature $\theta$, matter flux potential $\Omega$, and magnetic field potentials $\Phi$ and $\Phi_{\mathrm{e}}$ :

$$
\begin{gathered}
\left(\mathrm{D}^{2}-k^{2}\right)\left(\mathrm{D}^{2}-k^{2}-\lambda / P r\right) W-k^{2} R m G\left[\Omega-\left(\Psi+\xi \mathcal{L}^{\prime} / \mathcal{L}\right) \theta+\sigma \mathrm{D} \Phi\right]=0 \\
\left(\mathrm{D}^{2}-k^{2}-\lambda\right) \theta-W=0 \\
L e\left(\mathrm{D}^{2}-k^{2}-\lambda / L e\right) \Omega+\lambda \Psi \theta-(\sigma-\hat{\mu}) \lambda \mathrm{D} \Phi+\Psi_{\mathrm{H}} W=0 \\
\left(\sigma \mathrm{D}^{2}-\mu k^{2}\right) \Phi=-\mathrm{D}\left[\Omega-\left(\Psi+\xi \mathcal{L}^{\prime} / \mathcal{L}\right) \theta\right], \quad\left(\mathrm{D}^{2}-k^{2}\right) \Phi_{\mathrm{e}}=0
\end{gathered}
$$

\section{Boundary Conditions}

To completely specify solutions of Eqs. (3.12), one needs ten boundary conditions at confined surfaces with the addition of two conditions on $\Phi_{\mathrm{e}}$ far from the layer.

For the case of rigid boundary surfaces whose heat conductivity is very high in comparison with the enclosed ferrofluid, the boundary conditions on velocity and temperature read:

$$
W=\mathrm{D} W=\theta=0 \quad \text { at } \quad z= \pm 1 / 2 .
$$

It is natural to assume such boundaries to be at the same time completely impervious. The latter condition is expressed by setting the matter flux perturbation equal to zero,

$$
\mathrm{D} \Omega=0 \quad \text { at } \quad z= \pm 1 / 2 .
$$

Boundary conditions on the magnetic potential $\Phi$ are pretty complicated by the

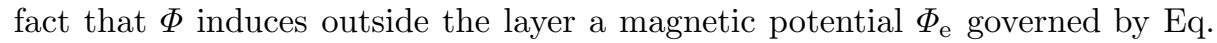


(3.12e). This equation have the solution $\Phi_{\mathrm{e}} \propto \exp ( \pm k z)$ which must decay far from the layer and be valid everywhere outside the layer including the layer boundaries too. Hence we come to the relations

$$
\mathrm{D} \Phi_{\mathrm{e}}=-k \Phi_{e} \quad \text { at } \quad z=1 / 2 ; \quad \mathrm{D} \Phi_{\mathrm{e}}=k \Phi_{e} \quad \text { at } \quad z=-1 / 2 .
$$

Boundary conditions of continuity of the tangential components of magnetic field and the normal component of magnetic induction on the layer boundaries have the form

$$
\partial \Phi / \partial x=\partial \Phi_{e} / \partial x, \quad \partial \Phi / \partial y=\partial \Phi_{e} / \partial y
$$

and

$$
\mathrm{D} \Phi+4 \pi\left(\frac{\partial M}{\partial \phi} \varphi+\frac{\partial M}{\partial T} \theta+\frac{\partial M}{\partial H} \mathrm{D} \Phi\right)=\mathrm{D} \Phi_{\mathrm{e}} .
$$

The conditions (4.4) are satisfied if

$$
\Phi=\Phi_{\mathrm{e}} \quad \text { at } \quad z= \pm 1 / 2
$$

whereas Eq. (4.5) on the substitution $M=M(\phi, T, \xi)$ takes the dimensionless form

$$
\sigma \mathrm{D} \Phi+\Omega-\left(\Psi+\frac{\xi \mathcal{L}^{\prime}}{\mathcal{L}}\right) \theta=\mathrm{D} \Phi_{\mathrm{e}} \quad \text { at } \quad z= \pm 1 / 2 .
$$

Relations (4.3) and (4.6) permit to write down: $\mathrm{D} \Phi_{\mathrm{e}}=\mp k \Phi$ at $z= \pm 1 / 2$, that gives us an opportunity to eliminate $\mathrm{D} \Phi_{\mathrm{e}}$ from (4.7). Taking into account the boundary conditions on the temperature (4.1), we obtain, finally, the closed boundary conditions on $\Phi$ :

$$
\sigma \mathrm{D} \Phi+k \Phi=-\Omega \quad \text { at } \quad z=1 / 2 ; \quad \sigma \mathrm{D} \Phi-k \Phi=-\Omega \quad \text { at } \quad z=-1 / 2 .
$$

Thus, there is no necessity at all to find the magnetic potential outside the layer. The relations (4.1), (4.2), and (4.8) represent the complete set of the rather realistic boundary conditions for the set of equations $(3.12 \mathrm{a})-(3.12 \mathrm{~d})$.

Below, however, we consider the case of idealized boundary conditions

$$
W=\mathrm{D}^{2} W=\theta=\Omega=\mathrm{D} \Phi=0 \quad \text { at } \quad z= \pm 1 / 2,
$$

which would be true for free, perfectly heat conducting and pervious boundaries made of a superconductor. In the latter case, magnetic field perturbations do not penetrate into the bulk of constrained horizontal plates, so that $\mathrm{D} \Phi_{\mathrm{e}}=0$. Thus, as it follows from (4.7) with allowance for the boundary conditions (4.9) on $\theta$ and $\Omega$, the value $\mathrm{D} \Phi$ at $z= \pm 1 / 2$ appears to be zero too.

\section{Exact Solution for Free Boundaries}

In the case of boundary conditions (4.9) there exists an analytical solution of Eqs. (3.12a) - (3.12d). Satisfying the boundary conditions and the equations by means of

$$
W \propto \theta \propto \Omega \propto \cos \pi z, \quad \Phi \propto \sin \pi z,
$$


we arrive at the characteristic equation for increments $\lambda$,

$$
\begin{aligned}
& \left(\pi^{2}+k^{2}+\lambda\right)\left(\pi^{2}+k^{2}+\lambda / \operatorname{Pr}\right)\left(\pi^{2}+k^{2}\right)\left[\lambda\left(\alpha \pi^{2}+k^{2}\right)+\operatorname{Le}\left(\beta \pi^{2}+k^{2}\right)\left(\pi^{2}+k^{2}\right)\right] \\
& -k^{4} \operatorname{Rm} G\left[\lambda\left(\xi \mathcal{L}^{\prime} / \mathcal{L}\right)+\left(\pi^{2}+k^{2}+\lambda\right) \Psi_{\mathrm{H}}+\operatorname{Le}\left(\pi^{2}+k^{2}\right)\left(\Psi+\xi \mathcal{L}^{\prime} / \mathcal{L}\right)\right]=0, \quad(5.1)
\end{aligned}
$$

where $\alpha=\hat{\mu} / \mu$ and $\beta=\sigma / \mu$. For stationary convective instability $(\lambda=0)$ the magnetic Rayleigh number is given by

$$
R m^{\mathrm{st}}=\frac{L e\left(\beta \pi^{2}+k^{2}\right)\left(\pi^{2}+k^{2}\right)^{3}}{k^{4} G\left[\Psi_{\mathrm{H}}+L e\left(\Psi+\xi \mathcal{L}^{\prime} / \mathcal{L}\right)\right]} .
$$

As it mentioned above, magnetic colloids are characterized by extremely small Lewis numbers (typically $L e \sim 10^{-4}$ ). Therefore, using the definitions (2.10) and (3.11b), one may rewrite the last equation in the form

$$
R m^{\mathrm{st}}=\frac{\operatorname{Le} \sigma^{2}\left(\beta \pi^{2}+k^{2}\right)\left(\pi^{2}+k^{2}\right)^{3}}{4 \pi \hat{\mu} k^{4} \mathcal{L}^{2}\left(\Psi+\xi \mathcal{L}^{\prime} / \mathcal{L}\right)[\Psi-(\hat{\mu}-1) \xi \mathcal{L} / \hat{\mu}]} .
$$

The magnetic Rayleigh number according to its definition (3.12a) is always positive. Therefore, as it seen from (5.2), stationary instability sets in only outside the interval

$$
-\frac{\xi \mathcal{L}^{\prime}(\xi)}{\mathcal{L}(\xi)}<\Psi<\frac{\hat{\mu}-1}{\hat{\mu}} \xi \mathcal{L}(\xi)
$$

i.e., either for a large enough positive separation ratio or for a sufficiently small negative $\Psi$. At the same time, as it will be shown below, inside the interval it can occur only oscillatory convective instability. In particular, the oscillatory instability is only possible in the absence of the Soret effect, i.e., when $\Psi=0$.

Omitting in (5.1) small terms with the coefficient $L e$, we obtain the cubic equation for $\lambda$ :

$$
\begin{gathered}
\left(\alpha \pi^{2}+k^{2}\right)\left(\pi^{2}+k^{2}\right) \lambda^{3}+(\operatorname{Pr}+1)\left(\alpha \pi^{2}+k^{2}\right)\left(\pi^{2}+k^{2}\right)^{2} \lambda^{2} \\
+\left[\left(\alpha \pi^{2}+k^{2}\right)\left(\pi^{2}+k^{2}\right)^{3}-k^{4} \operatorname{Rm} G\left(\Psi_{\mathrm{H}}+\xi \mathcal{L}^{\prime} / \mathcal{L}\right)\right] \operatorname{Pr} \lambda \\
=k^{4}\left(\pi^{2}+k^{2}\right) \operatorname{Pr} \operatorname{Rm} G \Psi_{\mathrm{H}} .
\end{gathered}
$$

Substituting in this equation $\lambda=\mathrm{i} \omega$ we arrive at two relations which determine the magnetic Rayleigh number for oscillatory instability,

$$
R m^{\mathrm{osc}}=\frac{(\operatorname{Pr}+1) \sigma^{2}\left(\alpha \pi^{2}+k^{2}\right)\left(\pi^{2}+k^{2}\right)^{3}}{4 \pi \hat{\mu} \operatorname{Pr} k^{4} \mathcal{L}^{2}\left(\Psi+\xi \mathcal{L}^{\prime} / \mathcal{L}\right)\left[\Psi+\left(\xi \mathcal{L}^{\prime} / \mathcal{L}\right)(1+\sigma / \hat{\mu} \operatorname{Pr})\right]},
$$

and the frequency of neutral convective oscillations,

$$
\omega^{2}=-\frac{[\Psi-(\hat{\mu}-1) \xi \mathcal{L} / \hat{\mu}]\left(\pi^{2}+k^{2}\right)^{2}}{\Psi+\left(\xi \mathcal{L}^{\prime} / \mathcal{L}\right)(1+\sigma / \hat{\mu} \operatorname{Pr})} .
$$

Since $R m^{\text {osc }}$ and $\omega^{2}$ must be both positive, the oscillatory instability can occur only if $\Psi$ lies within the interval (5.3). The corridor of oscillatory instability for 
a ferrofluid with the initial magnetic permeability $\mu_{0}=3$ is plotted in Fig. 1 for a wide enough range of the Langevin parameter $\xi$. On the upper border of the corridor, the frequency of convective oscillations turns into zero, whereas on its lower border the critical Rayleigh number turns into infinity. In the absence of magnetophoresis the corridor grows narrow so that its upper border passes along the line $\Psi=0$. In this case the oscillatory instability would be possible owing to the Soret effect only for $\Psi<0$. At last, if neither magnetophoresis nor Soret effect are in operation (i.e., no particle diffusion), then the cubic equation (5.4) for $\lambda$ is reduced to the square one,

$$
\begin{gathered}
\hat{\mu}\left(\alpha \pi^{2}+k^{2}\right)\left(\pi^{2}+k^{2}\right)\left[\lambda^{2}+(\operatorname{Pr}+1)\left(\pi^{2}+k^{2}\right) \lambda+\operatorname{Pr}\left(\pi^{2}+k^{2}\right)^{2}\right] \\
=4 \pi \operatorname{Pr} k^{4} \operatorname{Rm}\left(\xi \mathcal{L}^{\prime}\right)^{2} .
\end{gathered}
$$

This equation has no solutions of kind of $\lambda=i \omega$. Thus, if there is no diffusion, then there is no oscillatory instability as well. For stationary instability Eq. (5.7) gives

$$
R m^{(0)}=\frac{\hat{\mu}\left(\alpha \pi^{2}+k^{2}\right)\left(\pi^{2}+k^{2}\right)^{3}}{4 \pi k^{4}\left(\xi \mathcal{L}^{\prime}\right)^{2}} .
$$

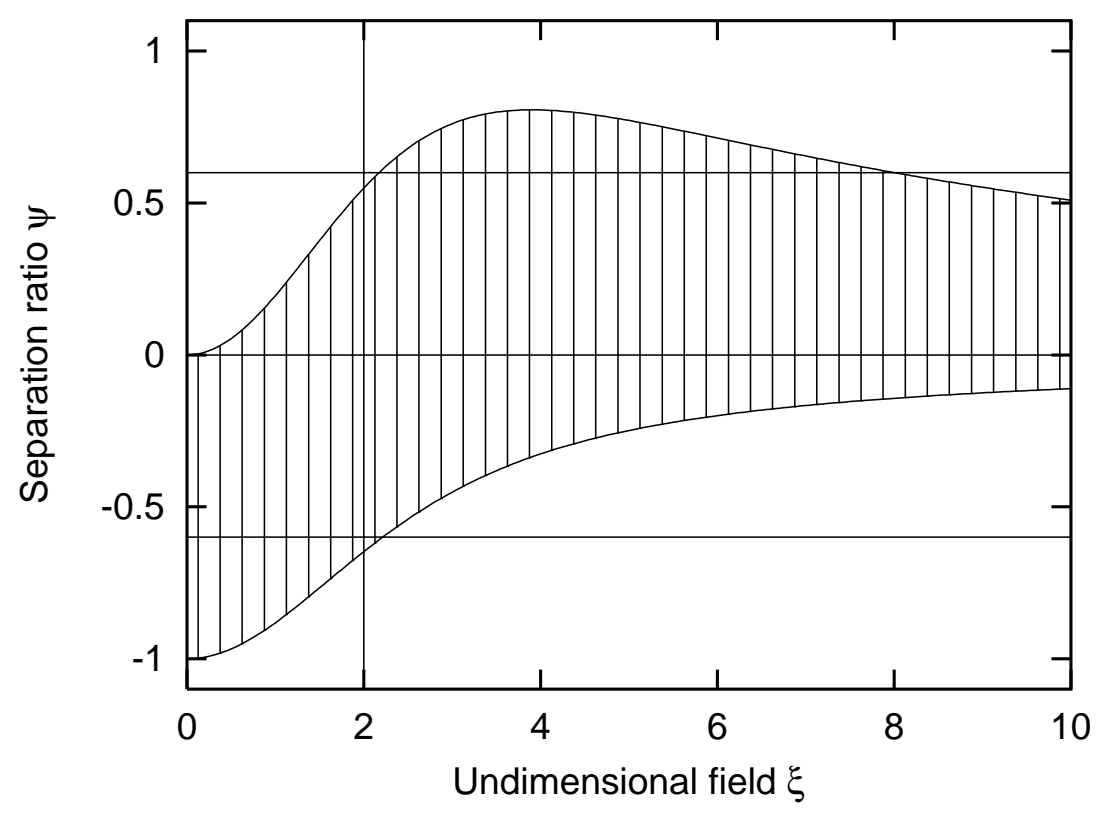

Fig. 1. The corridor of oscillatory instability (dashed) in $(\xi, \Psi)$ plane. Straight lines correspond to $\Psi=0, \Psi=-0.6, \Psi=+0.6$ and $\xi=2$. Cross sections of the surface $R m_{\mathrm{c}}(\xi, \Psi)$ along these lines are presented below in Figs. 2-5 respectively.

Below we shall consider $R m$ as a function of $\xi$ and $\Psi$. Any pair of fixed magnitudes of the parameters determines a neutral stability curve in the $(R m, k)$ 
plane. The minima of these curves, $R m_{\mathrm{c}}$, and the critical circular frequency of convective oscillations, $f=\omega_{\mathrm{c}} / 2 \pi$, are presented in Figs. 2-5. Figures 2-4 show $R m_{\mathrm{c}}$ and $f$ as functions of $\xi$ for three magnitudes of $\Psi$ marked in Fig. 1 by horizontal lines $\Psi=0, \Psi=-0.6$, and $\Psi=+0.6$. Figure 5 represents a stability diagram in the plane $\left(R m_{\mathrm{c}}, \Psi\right)$ for the magnitude $\xi=2$ marked in Fig. 1 by the vertical line.

\section{Discussion of Results and Conclusion}

At first, let us consider the case $\Psi=0$. Then Eqs. (5.5)-(5.6) become somewhat simpler:

$$
\begin{aligned}
R m^{\mathrm{osc}} & =\frac{(\operatorname{Pr}+1) \sigma^{2}\left(\alpha \pi^{2}+k^{2}\right)\left(\pi^{2}+k^{2}\right)^{3}}{4 \pi k^{4}\left(\xi \mathcal{L}^{\prime}\right)^{2}(\operatorname{Pr} \hat{\mu}+\sigma)}, \\
\omega^{2} & =\frac{3 \operatorname{Pr}\left(\mu_{0}-1\right) \mathcal{L}^{2}\left(\pi^{2}+k^{2}\right)^{2}}{\operatorname{Pr} \hat{\mu}+\sigma} .
\end{aligned}
$$

The function $R m^{\text {osc }}(k)$ has a minimum $R m_{\mathrm{c}}^{\text {osc }}$ at the critical wave number $k_{\mathrm{c}}$ determined by the formula

$$
k_{\mathrm{c}}^{2}=(\pi / 2)^{2}\left(1-\alpha+\sqrt{1+14 \alpha+\alpha^{2}}\right) .
$$

Here $\alpha=\hat{\mu} / \mu \leq 1$ depends on the dimensionless magnetic field $\xi$ and the initial magnetic permeability $\mu_{0}$. For commonly used ferrofluids (with $1<\mu_{0}<5$ ) $k_{\mathrm{c}}$ weakly depends on $\xi$. For instance, at $\mu_{0}=3$ the critical wave number lies inside the interval $2.97<k_{\mathrm{c}}<3.14$ for any $\xi$, so that $k_{\mathrm{c}}$ variations do not exceed $6 \%$.

Substituting $k_{\mathrm{c}}^{2}$ from (6.2) into (6.1) we find the critical Rayleigh number and the frequency of neutral oscillations. The values $\log R m_{\mathrm{c}}^{\mathrm{osc}}$ and $f=\omega_{\mathrm{c}} / 2 \pi$ as functions of $\xi$ are presented in Fig. 2. In the same figure it is shown the logarithm of $R m_{\mathrm{c}}^{(0)}$ determined by Eq. (5.8) under the replacement $k^{2}$ by $k_{\mathrm{c}}^{2}$ from (6.2).

We dare say that both the critical Rayleigh numbers, $R m_{\mathrm{c}}^{\text {osc }}$ and $R m_{\mathrm{c}}^{(0)}$ have a direct physical meaning. Depending on conditions of a real experiment it will set in either stationary convection above $R m_{\mathrm{c}}^{(0)}$ or oscillatory convection above $R m_{\mathrm{c}}^{\text {osc }}$. The point is that ferrofluids are characterized by two very different time scales [13]. One of two is the above-mentioned long mass diffusion time $\tau_{\mathrm{D}}=d^{2} / \pi^{2} D$ : even in a thin layer $(\sim 1 \mathrm{~mm})$ the concentration equilibrium is established for a time longer than one hour. Another one is a short thermodiffusion time $\tau_{\mathrm{T}}=d^{2} / \pi^{2} \kappa$ : in the same fluid layer the stationary temperature gradient sets in merely in one second. The ratio $\tau_{\mathrm{T}} / \tau_{\mathrm{D}}$ is equal just to the Lewis number $L e=D / \kappa \sim 10^{-4}$. The presence of such different time scales makes possible two scenarios of the onset of convection. First, the temperature difference between the layer boundaries can be increased from zero till a supercritical value during a time $\tau \ll \tau_{D}$. Then diffusion processes have no time to evolve, so the magnetic colloid behaves like a pure fluid. Indeed, magnetic grains do not move without diffusion relative to the surrounding liquid matrix, they are "frozen" into it. Just 


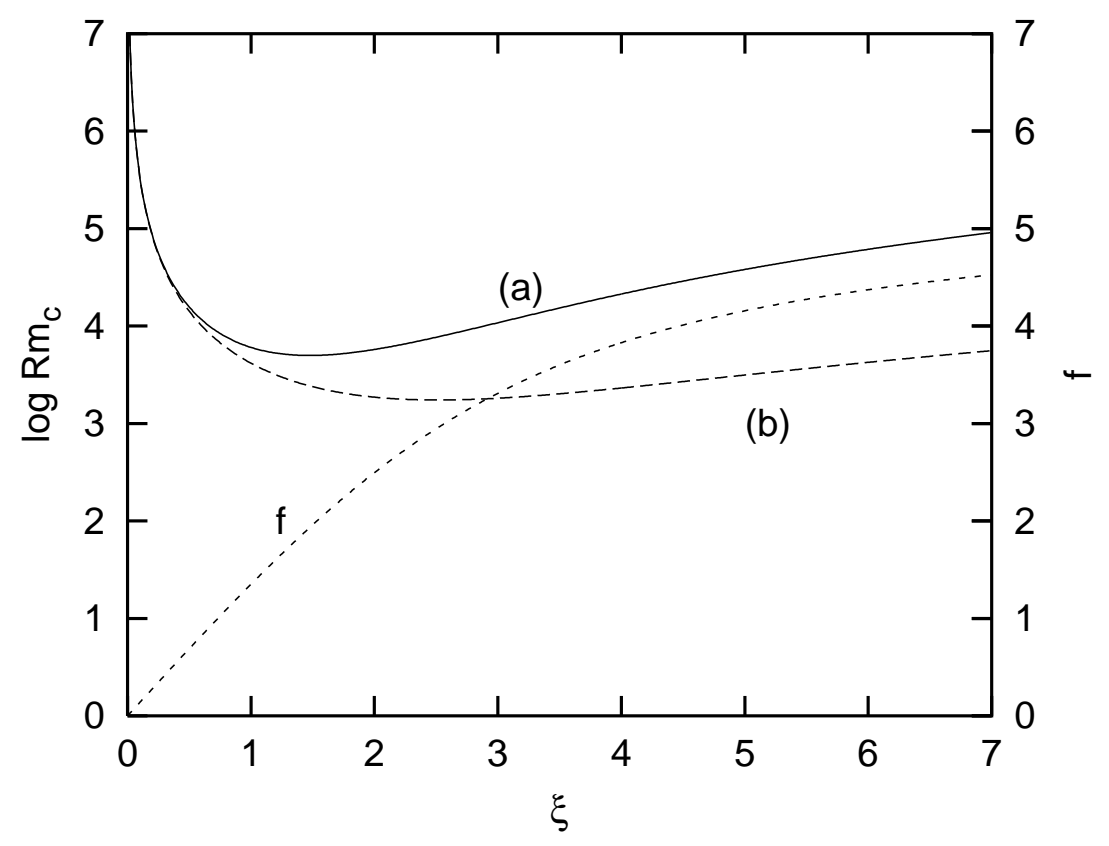

Fig. 2. Critical magnetic Rayleigh number $R m_{\mathrm{c}}$ and circular frequency of neutral oscillations $f$ versus magnetic field strength $\xi$. Curve $(a)-$ oscillatory solution $R m_{\mathrm{c}}^{\text {osc }}$ obtained with allowance for magnetophoresis but without Soret effect $(\Psi=0)$, curve (b) - stationary solution $R m_{\mathrm{c}}^{(0)}$ in the absence of the particle diffusion (i.e., there are neither magnetophoresis nor the Soret effect).

that very case has been studied in [3-8]. Stationary convection starts in this case at $R m_{\mathrm{c}}^{(0)}$. The latter value has a minimum at a certain $\xi$; for a water-based ferrofluid $(\operatorname{Pr}=7)$ with $\mu_{0}=3$ this minimum, $\min \left[R m_{\mathrm{c}}^{(0)}\right]=1742$, is reached at $\xi=2.52$ and $k_{\mathrm{c}}=3.02$.

Second scenario can be realized in a thin enough fluid layer, where the long diffusion time, $\tau_{\mathrm{D}}$, is not too long. Then it is possible to adjust so low heating rate, that the temperature difference across the layer, $\Delta T$, will be formed for a time $\tau>\tau_{\mathrm{D}}$. Under this condition the concentration gradient induced by the temperature gradient due to magnetophoresis (the Soret effect is absent at $\Psi=0$ ) will be built up undisturbed by convection. In this case, as it shown above, there arises oscillatory instability at $R m_{\mathrm{c}}^{\text {osc }}$. For a ferrofluid under consideration the critical Rayleigh number reaches the minimum, $\min \left[R m_{\mathrm{c}}^{\text {osc }}\right]=4993$, at $\xi=$ 1.47 ; corresponding wave number and frequency of neutral oscillations are $k_{\mathrm{c}}=$ 3.08 and $\omega_{\mathrm{c}}=12.13$. The frequency increases monotonously with $\xi$ approaching the saturation value $\omega_{\mathrm{c}}=34.19$ at $\xi \rightarrow \infty$.

Let us estimate the temperature difference and other parameters required to cause oscillatory convection by the thermomagnetic mechanism. Assuming the mean diameter of magnetite particles $\left(M_{\mathrm{s}}=480 \mathrm{G}\right)$ to be $2 a=11 \mathrm{~nm}$, we 
obtain the particle magnetic moment $m=\left(4 \pi a^{3} / 3\right) M_{\mathrm{s}}=3.345 \times 10^{-16} \mathrm{erg} / \mathrm{Oe}$, so the value $\mu_{0}=1+4 \pi \phi M_{\mathrm{s}} m / 3 k_{\mathrm{B}} T=3$ is reached at the room temperature at $\phi=12 \%$, and $\xi=m H / k_{\mathrm{B}} T=1$ at $H=122$ Oe. Thus the minimum in the curve of $\log R m_{\mathrm{c}}^{\mathrm{osc}}$ in Fig. 2 takes place at $H=180$ Oe (to get such a field within the ferrofluid layer one must apply externally $H_{e}=\mu_{0} H=540$ Oe). The minimum Rayleigh number $(\approx 5000)$ is reached at $(\Delta T)_{c}=(1.02 / d) \mathrm{K} / \mathrm{cm}$, thus the critical temperature difference is about $10 \mathrm{~K}$ in the layer of $d=1 \mathrm{~mm}$. For this layer the unit of time, $d^{2} / \kappa$, is equal to $6.9 \mathrm{~s}$, so the above-pointed dimensionless frequency $\omega_{\mathrm{c}}=12.13$ of critical convective oscillations corresponds to the period of $3.6 \mathrm{~s}$.

Maps of stability in the $\left(R m_{\mathrm{c}}, \xi\right)$-plane are presented in Figs. 3 and 4 for $\Psi=-0.6$ and $\Psi=+0.6$, respectively. As it seen in Fig. 1, for both these magnitudes of the separation ratio there are certain intervals of $\xi$ in which it can occur oscillatory instability. Out of the intervals there arises stationary Soret convection which is characterized by very small Rayleigh numbers (5.2) due to the smallness of the Lewis number (for a water-based ferrofluid $L e=1.9 \times 10^{-4}$ ). The critical Rayleigh number for stationary instability, $R m_{\mathrm{c}}^{\mathrm{st}}$, is obtained from (5.2) under the substitution $k_{\mathrm{c}}^{2}$ from (6.2) with the replacement of $\alpha$ by $\beta$.

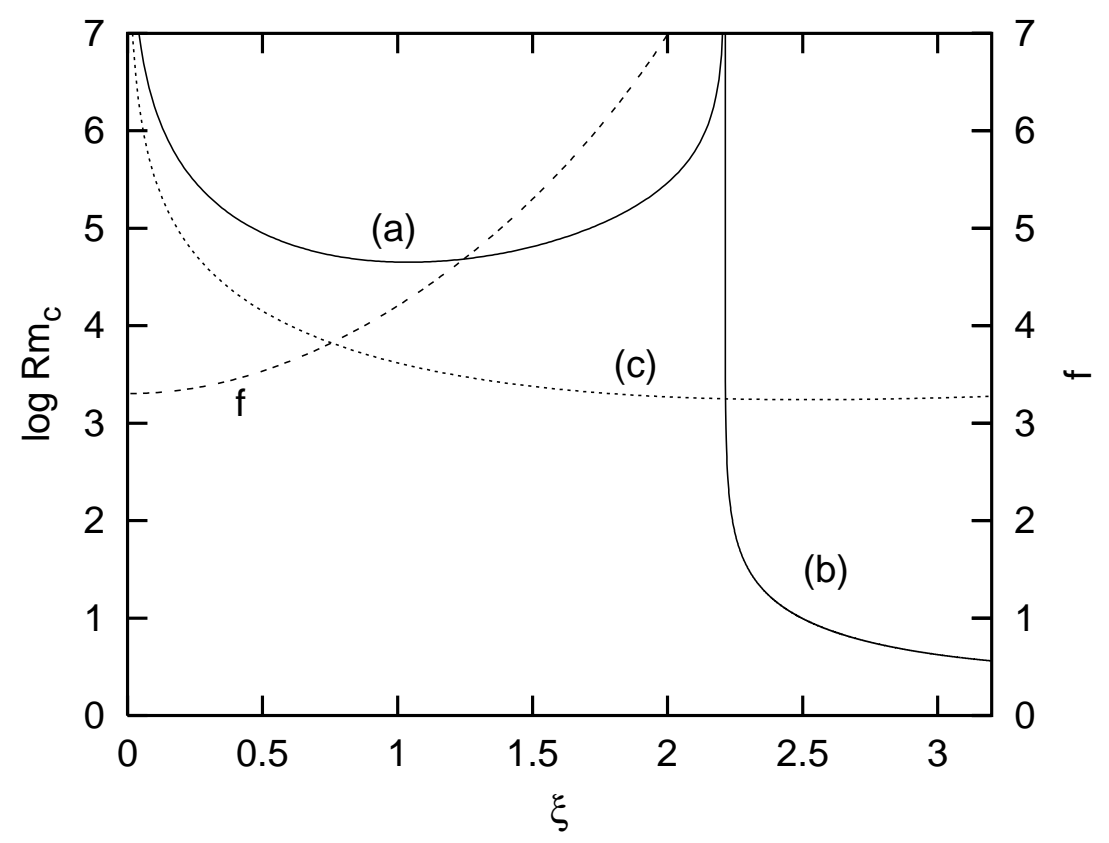

Fig. 3. Critical magnetic Rayleigh number and frequency of neutral oscillations versus magnetic field strength. Curve $(a)$ - oscillatory solution $R m_{\mathrm{c}}^{\text {osc }}$ and $(b)-$ stationary solution $R m_{\mathrm{c}}^{\text {st }}$ with allowance for magnetophoresis and the negative Soret effect $(\Psi=$ -0.6 ); curve $(c)$ - stationary solution $R m_{\mathrm{c}}^{(0)}$ in the absence of the particle diffusion. 


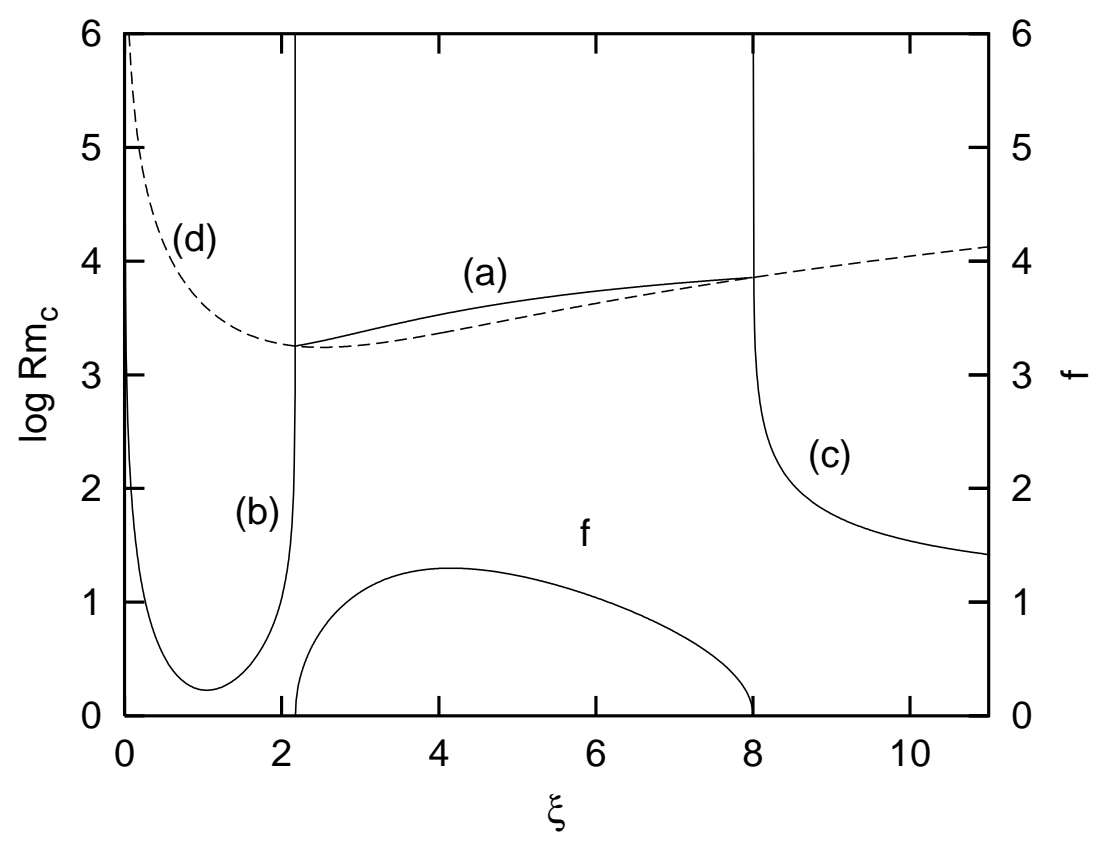

Fig. 4. Critical magnetic Rayleigh number and frequency of neutral oscillations versus magnetic field strength. Curve $(a)$ - oscillatory solution $R m_{\mathrm{c}}^{\mathrm{osc}}$ and $(b),(c)$ - stationary solution $R m_{\text {c }}^{\text {st }}$ with allowance for magnetophoresis and the positive Soret effect $(\Psi=$ $0.6)$; curve $(d)$ - stationary solution $R m_{\mathrm{c}}^{(0)}$ in the absence of the particle diffusion.

For a fixed negative separation ratio, $0>\Psi>-1$, oscillatory instability takes place within an interval of the field $0<\xi<\xi_{0}$ (see Figs. 1 and 3), where $\xi_{0}$ satisfies the equation

$$
\Psi+\xi \mathcal{L}^{\prime}(\xi) / \xi=0 .
$$

On the borders of the interval, the critical Rayleigh number (5.5) turns into infinity as $\xi^{-2}$ for $\xi \rightarrow 0$ and as $\left(\xi-\xi_{0}\right)^{-1}$ for $\xi \rightarrow \xi_{0}$ (see Fig. 3). The frequency of neutral oscillations, $\omega_{\mathrm{c}}(\xi)$, commences from a finite magnitude at $\xi=0$,

$$
\omega_{\mathrm{c}}(0)=2 \pi^{2} \sqrt{\frac{\operatorname{Pr}|\Psi|}{1+\operatorname{Pr}(1-|\Psi|)}},
$$

and grows monotonously with $\xi$ until it reaches $\xi_{0}$.

The upper border of the interval (5.3) is described by

$$
\Psi(\xi)=\frac{\hat{\mu}-1}{\hat{\mu}} \xi \mathcal{L}(\xi),
$$

where $\hat{\mu}=1+3\left(\mu_{0}-1\right) \mathcal{L}^{\prime}(\xi)$. The function (6.4) has a maximum $\Psi_{m}$ at a certain value $\xi_{m}$ dependent on the initial magnetic permeability $\mu_{0}=1+4 \pi \chi_{0}$. For the fluid under consideration $\left(\mu_{0}=3\right)$ we find $\xi_{m}=3.90, \Psi_{m}=0.806$. For any 
positive $\Psi<\Psi_{m}$ there exists an interval $\xi_{1}<\xi<\xi_{2}$ of oscillatory instability see Figs. 1 and 4 . The frequency of oscillations reaches a maximum within the interval and turns into zero on its borders. Oscillatory branch $(a)$ of $R m_{\mathrm{c}}(\xi)$ links in Fig. 4 two codimension-2 points, $R m_{\mathrm{c}}\left(\xi_{1}\right)$ and $R m_{\mathrm{c}}\left(\xi_{2}\right)$, in which stationary branches $(b$ and $c)$ bifurcate.

A diagram of stability in the $\left(R m_{\mathrm{c}}, \Psi\right)$-plane at the fixed magnitude of the field, $\xi=2$, is shown in Fig. 5. This diagram looks more or less traditional for binary mixtures $[14,15]$ with exception of two things. Firstly, the codimension-2 point (i.e., the intersection point of $a$ and $c$ branches) is usually located in the close vicinity of $\Psi=0$. In Fig. 5 this point has essentially shifted towards positive $\Psi$ due to magnetophoresis. Secondly, the stationary branch $b$ as a general rule is located in the region of negative Rayleigh numbers, i.e., the branch determines the onset of convection in the binary mixture heated from above. Thermomagnetic mechanism of convection knows, however, neither the top nor the bottom: the magnetic Rayleigh number (3.11a) is proportional to $(\Delta T)^{2}$, so it is always positive.

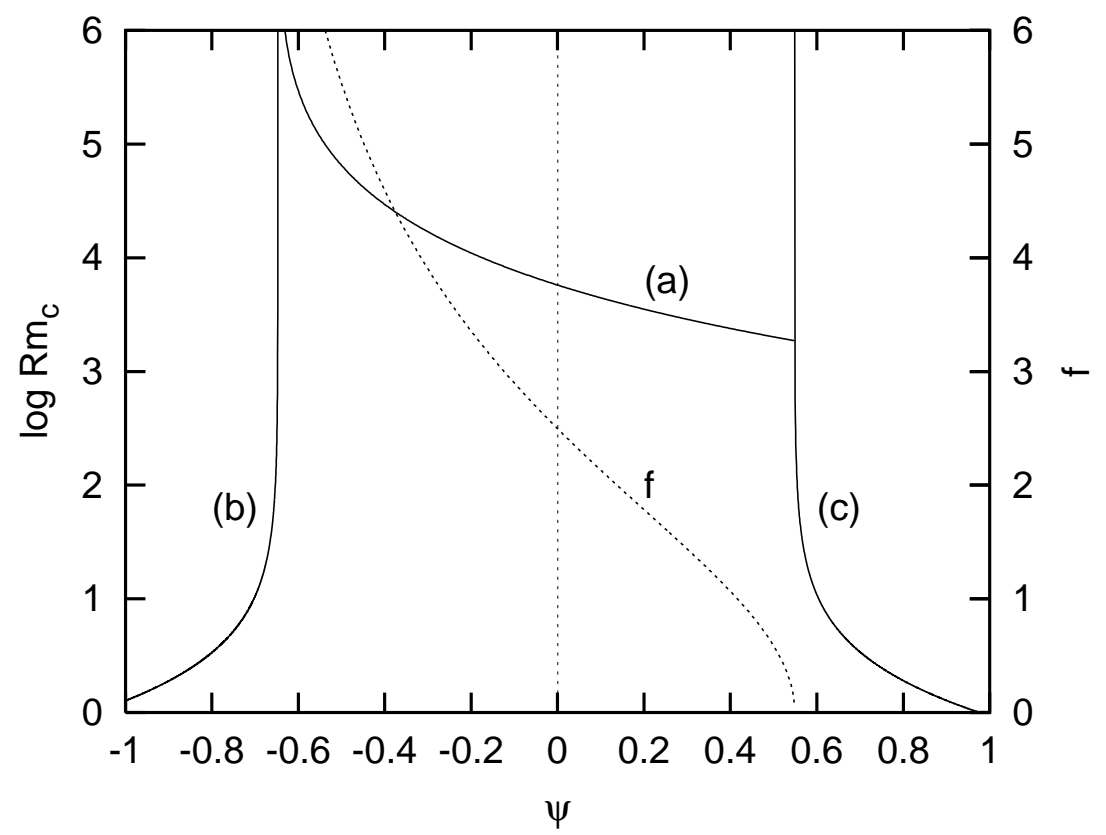

Fig. 5. Critical magnetic Rayleigh number and frequency of neutral oscillations versus separation ratio at the fixed magnetic field strength $\xi=2$. Curve $(a)-$ oscillatory instability, curves $(b)$ and $(c)$ - stationary instability.

Thus, convective instability of magnetized ferrofluids is strongly effected by the magnetophoretic and thermophoretic (the Soret effect) transfer of magnetic grains. The most interesting consequence of the transfer is an opportunity to ob- 


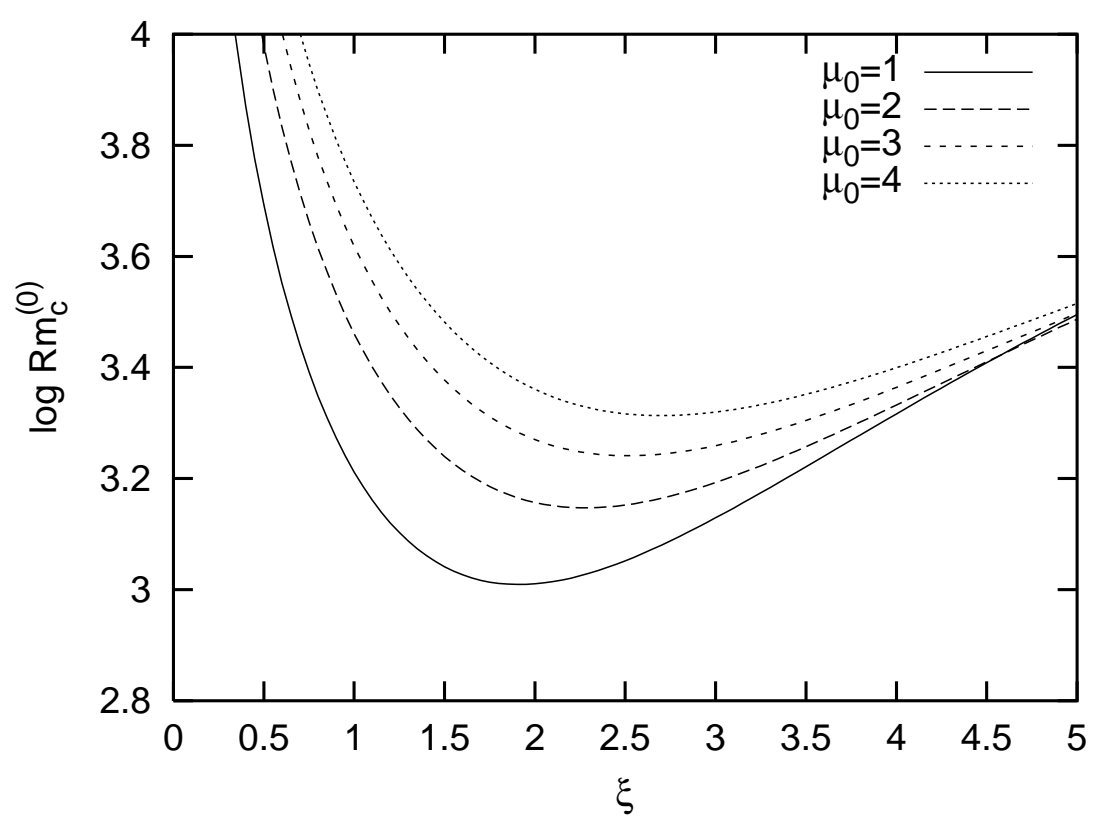

Fig. 6. Stationary instability curves for different $\mu_{0}$ in the case when the particle diffusion is not operative.

serve oscillatory convective instability. However, as we have already commented, to observe predicted oscillations one needs to satisfy certain experimental conditions. Namely, the temperature difference must not be increased faster than the limit imposed by concentration diffusion. In the opposite case, a ferrofluid behaves like a pure fluid what results in stationary instability at the critical Rayleigh number $R m_{\mathrm{c}}^{(0)}$; its dependence on $\xi$ for some magnitudes of $\mu_{0}$ is shown in Fig. 6. In conclusion, it is worth to remind that all our results are relevant to thin ferrofluid layers $(\sim 1 \mathrm{~mm})$ where magnetic mechanism of convection predominates over the buoyancy mechanism, and the characteristic diffusion time, $\tau_{\mathrm{D}}$, is not too long. To compare above-stated theoretical predictions with future experimental results one should perform a more precise analysis, which would satisfy realistic boundary conditions (4.1), (4.2) and (4.8) instead of idealized boundary conditions (4.9) used in the paper.

\section{Acknowledgments}

I am grateful to the Alexander von Humboldt Foundation for a Meitner-Humboldt research award and acknowledge the hospitality at the Bayreuth and Saarlandes Universities. This work has been also supported by a Grant 336/00-15.3 from the Israel Science Foundation. 


\section{References}

1. M.I. Shliomis: Sov. Phys.-Uspekhi 17, 153 (1974)

2. R.E. Rosensweig: Ferrohydrodynamics (Cambridge University Press, Cambridge 1985)

3. V.M. Zä̌tsev, M.I. Shliomis: J. Appl. Mech. Techn. Phys. 9, 24 (1968)

4. B.A. Finlayson: J. Fluid Mech. 40, 753 (1970)

5. M.I. Shliomis: Fluid Dynamics 6, 957 (1973)

6. P.J. Stiles, M. Kagan: J. Magn. Magn. Mater. 85, 196 (1990)

7. C.L. Russell, P.J. Blennerhassett, P.J. Stiles: J. Magn. Magn. Mater. 149, 119 (1995)

8. A. Recktenwald, M. Lücke: J. Magn. Magn. Mater. 188, 326 (1998)

9. J.-C. Bacri: private communication

10. M.I. Shliomis: Sov. Phys.-JETP 34, 1291 (1972)

11. B.V. Derjaguin, S.S. Dukhin, A.A. Korotkova: Kolloidn. Zh. 23, 53 (1961)

12. L.D. Landau, E.M. Lifshitz: Electrodynamics of Continuous Media (Pergamon Press, New York 1984)

13. M.I. Shliomis, M. Souhar: Europhys. Lett. 49, 55 (2000)

14. D.T.J. Hurle, E. Jakeman: J. Fluid. Mech. 47, 667 (1971)

15. E. Knobloch, D.R. Moore: Phys. Rev. A 37, 860 (1988) 


\section{Glossary}

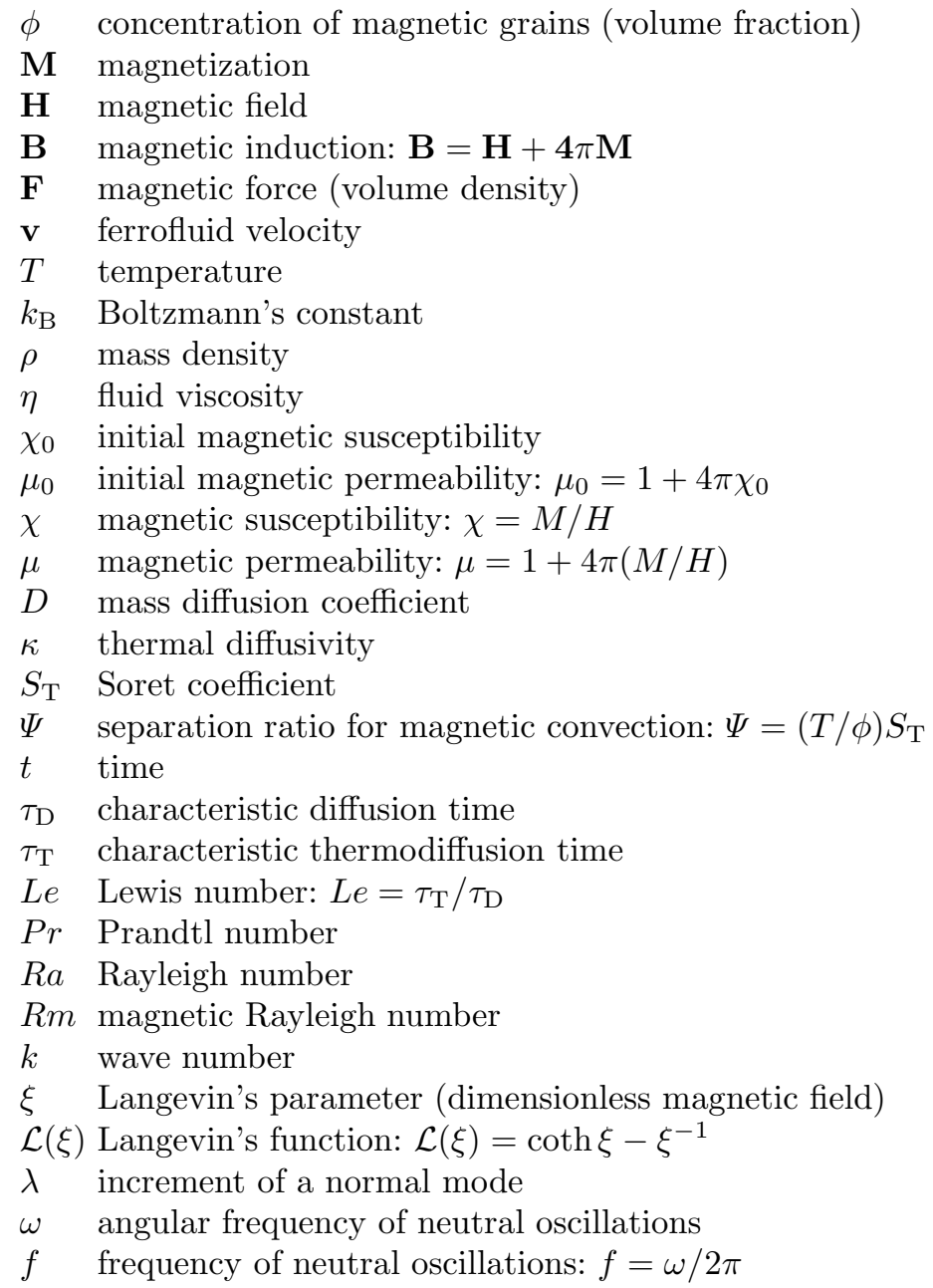

